\title{
Study of impulse voltage distribution in transformer windings
}

\begin{abstract}
In this paper, analysis impulse voltage distribution in transformer winding, and winding of the voltage impulse response with a standard full wave and clipped wave especially at the ends of the entanglement presented. Compared with experimental data EMTPDC simulation, the result of $t<1.0 \varepsilon$ s voltage difference occurs. For $t>2.0 \varepsilon s$ was obtained near the same voltage large, between experimental and simulation results. So we can say that circuit simulation can mimic the real situation EMTDC/PSCAD. Construction of turns and the thickness of insulation ( $\mathrm{Cs}$ and $\mathrm{Cg}$ ) transformer, poorer have considerable effect on the distribution center of experiencing transient voltage transformer (transitional-symptoms). Winding section closest to the high voltage high voltage gradient than most parts of another entanglement, tests were performed in a standard form according to the current surge of IEC$718 / 20 \varepsilon s$. Important information from this study is that the simulation gives a more pessimistic outcome (EMTDC/PSCAD calculations show a larger stress values compared with experimental). Although it is difficult to imitate the behavior of entanglement in the initial transient, but the real situation is not worse. Thus, this simulation can be used as EMTPDC experimental substitution want to know the response to the current surge of turns.
\end{abstract}

Keyword: EMTDC/PSCAD; Transformer model; Transient overvoltage 